\title{
Cosmic Steps in Modeling Dark Energy
}

\author{
Tower Wang* \\ Center for High-Energy Physics, Peking University, \\ Beijing 100871, China
}

(Dated: July 28, 2021)

\begin{abstract}
The past and recent data analyses gave some hints of steps in dark energy. Considering the dark energy as a dynamical scalar field, we investigate several models with various steps: a step in the scalar potential, a step in the kinetic term, a step in the energy density and a step in the equation-of-state parameter $w$. These toy models provide a workable mechanism to generate steps and features of dark energy. Remarkably, a single real scalar can cross $w=-1$ dynamically with a step in the kinetic term.
\end{abstract}

PACS numbers: 95.36.+x, 98.80.-k

\section{INTRODUCTION}

Dark energy is a popular explanation for the recent acceleration of our Universe. In order to draw a portrait of the dark energy, it is necessary to parameterize it and constrain the parameters from observational data. By the past and recent data analyses, it was indicated that the dark energy could have various steps in its parameters. Several years ago, a late-time transition in the equation of state (EOS) was studied by Bassett et al [1]. Very recently, Huang et al [2] reported that the dark energy might spring out at a low redshift. However, there were few theoretical studies to explain such steps in the EOS or in the density of dark energy.

Our purpose here is to make a step toward this direction. We will treat the dark energy as a dynamical scalar field, and then study a step in the scalar potential, in the kinetic term, in the energy density and in the EOS respectively. Lacking of enough observational data, hitherto the portrait of dark energy is still vague and the hints of steps are weak, so we do not attempt to build a realistic model in this paper. Instead, to give a vivid picture of the mechanism, we will play with simplified toy models and choose exaggerated model parameters. Because these models are difficult to solve analytically, for the most time we will rely on numerical algorithms. The models and algorithms can be easily extended and refined to give a more realistic description of the dark energy.

Although this could be the first time to systematically study cosmic steps in dark energy models with explicit Lagrangian, the stepped model is not novel in cosmology. It is an old story: possibly steps of the inflaton potential have left some fingerprints at the born time of our Universe [3]. Since the dark energy is more elusive, the

\footnotetext{
*Electronic address: wangtao218@pku.edu.cn
}

physical origin of the stepped dark energy field is less clear than the stepped inflaton field.

\section{METHODOLOGY}

Before going to specific models, we will describe the general framework and numerical methods in some detail. Impatient readers can skip directly to the next section to get our models (10), (13), (20) and main results depicted in figures.

In the absence of spatial fluctuations, the Lagrangian density of a scalar field minimally coupled to gravity has the form

$$
\mathcal{L}_{\phi}=a^{3}\left[\frac{1}{2} f(\phi) \dot{\phi}^{2}-V(\phi)\right],
$$

where $a$ is the scale factor. Here the function $f(\phi)$ in the kinetic term is new. It is positive for quintessence [4] and negative for phantom [5]. Later on we will also discuss a new model in which $f(\phi)$ evolves dynamically from +1 to -1 . In that situation, the single real scalar plays the role of quintom [6].

In a flat universe dominated by dark energy together with cold dark matter, if we ignore the contribution of ordinary matter for simplicity, then the evolution dynamics is governed by the following Friedmann equations

$$
\begin{aligned}
H^{2} & =\frac{8 \pi G_{N}}{3}\left(\rho_{m}+\rho\right) \\
& =\frac{8 \pi G_{N}}{3}\left[\frac{\rho_{m 0} a_{0}^{3}}{a^{3}}+\frac{1}{2} f(\phi) \dot{\phi}^{2}+V(\phi)\right], \\
\dot{H} & =-4 \pi G_{N}\left(\rho_{m}+\rho+p\right) \\
& =-4 \pi G_{N}\left[\frac{\rho_{m 0} a_{0}^{3}}{a^{3}}+f(\phi) \dot{\phi}^{2}\right] .
\end{aligned}
$$

Here $\rho_{m}$ is the energy density of dark matter, taking value $\rho_{m 0}$ at the present time with the scale factor $a=a_{0}$. The 
dark energy $\phi$ has an energy density $\rho$ and a pressure $p$, whose subscripts have been left out for briefness. One should not mistake $\rho$ and $p$ as the total energy density and the total pressure. The EOS parameter is defined by $w=p / \rho$ as usual.

In the above we have used a dot to denote the derivative with respect to comoving (physical) time $t$. For instance, we have taken the convention of notation $\dot{\phi}=$ $d \phi / d t$ and defined $H=\dot{a} / a$. It will be convenient to employ the notations $x=\ln \left(a / a_{0}\right)=-\ln (1+z)$ and $\phi^{\prime}=d \phi / d x$, then they give us a useful relation $\dot{\phi}=H \phi^{\prime}=-H(1+z) \phi_{, z}$. Utilizing (2), we find the kinetic energy is

$$
\frac{1}{2} f \dot{\phi}^{2}=\frac{8 \pi G_{N} f\left[\rho_{m 0}(1+z)^{3}+V\right](1+z)^{2} \phi_{, z}^{2}}{6-8 \pi G_{N} f(1+z)^{2} \phi_{, z}^{2}} .
$$

The equation of motion

$$
f \ddot{\phi}+3 H f \dot{\phi}+\frac{1}{2} f_{, \phi} \dot{\phi}^{2}+V_{, \phi}=0
$$

for the scalar field can be written as

$$
\begin{aligned}
& 4 \pi G_{N} f(1+z)\left[\rho_{m 0}(1+z)^{3}+4 V\right] \phi_{, z} \\
= & 8 \pi G_{N} f(1+z)^{2}\left[\rho_{m 0}(1+z)^{3}+V\right] \phi_{, z z} \\
& +16 \pi^{2} G_{N}^{2} f^{2}(1+z)^{3}\left[\rho_{m 0}(1+z)^{3}+2 V\right] \phi_{, z}^{3} \\
& +4 \pi G_{N} f_{, \phi}(1+z)^{2}\left[\rho_{m 0}(1+z)^{3}+V\right] \phi_{, z}^{2} \\
& +V_{, \phi}\left[3-4 \pi G_{N} f(1+z)^{2} \phi_{, z}^{2}\right] .
\end{aligned}
$$

Given explicit form of $f(\phi)$ and $V(\phi)$, equation (6) alone dictates the evolution of dark energy. But this is a highly nonlinear second order differential equation. In most cases we have to resort to numerical methods. We will take two slightly different schemes, dubbed the linear iteration method and the cubic iteration method, since they reform equation (6) into linear algebraic equations or cubic algebraic equations respectively. Let us elaborate on them now.

For numerically evolving equation (6) from an initial point $z_{i}$ to the final point $z_{f}$, we partition the interval $\left[z_{i}, z_{f}\right]$ into $N$ equal subintervals of width $h=z_{j}-z_{j-1}=$ $\left(z_{f}-z_{i}\right) / N$. It is convenient to notate $\phi_{j}=\phi\left(z_{j}\right)$ for short, where $j=0,1,2, \ldots, N$. For numerical computation, the derivatives can be approximated by finite differences

$$
\begin{aligned}
\left.\frac{d \phi}{d z}\right|_{z=z_{j}} & =\frac{\phi_{j+1}-\phi_{j-1}}{2 h} \\
\left.\frac{d^{2} \phi}{d z^{2}}\right|_{z=z_{j}} & =\frac{\phi_{j+2}-2 \phi_{j}+\phi_{j-2}}{4 h^{2}} .
\end{aligned}
$$

Making use of approximations (77) and (8), one can recast equation (6) into a linear algebraic equation with respect to $\phi_{j+2}$. Starting with the initial values of $\phi_{j}$ at $j=0,1,2,3$, we can get the values of all $\phi_{j}$ with $j>3$ iteratively. The linear iteration method gives a unique path of $\phi$ for numerical evolutions. Its disadvantage is the excessive number of initial conditions. Remember that for a second order differential equation we usually impose two initial conditions. In practical operation, we treat with potentials flat at the initial point $\phi_{i}$, and set $\phi_{0}=\phi_{1}=\phi_{2}=\phi_{3}=\phi_{i}$.

Rather than (8) one may tend to estimate the second order derivative with

$$
\left.\frac{d^{2} \phi}{d z^{2}}\right|_{z=z_{j}}=\frac{\phi_{j+1}-2 \phi_{j}+\phi_{j-1}}{h^{2}} .
$$

Now we need only two initial conditions, for instance, $\phi_{0}=\phi_{1}=\phi_{i}$. This is the starting point of the cubic iteration scheme. In such a scheme, instead of a linear equation of $\phi_{j+2}$, one has to solve a cubic equation with respect to $\phi_{j+1}$. The formula of roots of a cubic equation is well known. For our purpose, it is enough to treat them in two general categories: $(i)$ if the equation has one real root and a pair of imaginary roots, then $\phi_{j+1}$ takes a value of the real root; (ii) if all roots are real, we determine the value of $\phi_{j+1}$ by minimizing $\left|\phi_{j+1}-\phi_{j}\right|$. The trick enables us to pick out the smoothest evolution path of $\phi$, and fortunately this path is unique in our simulation. Even if the path is not unique after applying the above trick, one can still find the smoothest path by further minimizing $\left|\phi_{j+1}+\phi_{j-1}-2 \phi_{j}\right|$, etc.

The above two methods are operated independently in our algorithms. As double checks, they agree with each other very well. In fact, the resulting graphs look like duplicates. During the numerical simulation, we define the reduced Planck mass $M_{p l}=1 / \sqrt{8 \pi G_{N}}$, the fractional density of dark energy $\Omega_{\mathrm{DE}}=\rho_{\mathrm{DE}} /\left(3 M_{p}^{2} H^{2}\right)$ and the present fractional density of dark matter $\Omega_{m 0}=$ $\rho_{m 0} /\left(3 M_{p}^{2} H_{0}^{2}\right)$, where $H_{0}$ is Hubble parameter at the present time. Moreover, we set $\Omega_{m 0}=0.3$ and work in the unit $M_{p l}=1$. In a flat universe, ignoring the contribution of ordinary matter, one has $\Omega_{\mathrm{DE}}+\Omega_{m}=1$. For every specific model below, we evolve equation (6) from $z_{i}=20$ to $z_{f}=0$ with $N=2000$ subintervals. Fixing the initial conditions and other parameters, if we increase either $N$ or $z_{i}$, the change in results is unobservable. This confirms the reliability of our methods above and results below.

\section{MODELS WITH STEPS}

\section{A. Steps in the Scalar Potential}

So far we have not specified our models. It will be done in this section. Firstly, suppose the potential of dark energy is broadly flat but has a sudden transition near $\phi=b$. Then it is natural to model such a stepped potential in the following form

$$
V(\phi)=V_{0}\left[1+c \tanh \left(\frac{\phi-b}{d}\right)\right] .
$$



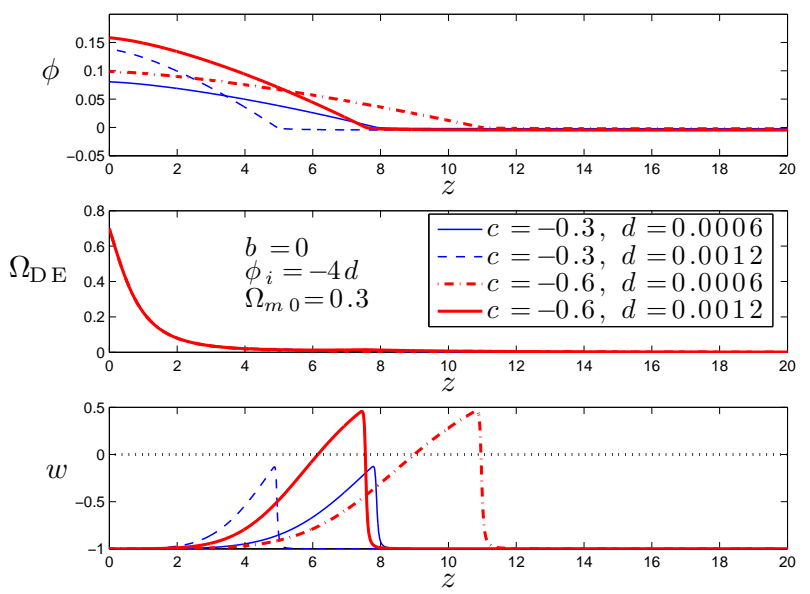

Figure 1: (color online). Evolution curves of the quintessence field with potential (10) and $f=+1$. There are bumps in the curves of fractional density and EOS parameter, although the bump in fractional density is almost unnoticeable. The amplitude and location of bumps can be tuned by changing parameters in our model. We set $8 \pi G_{N}=1$ in all figures.
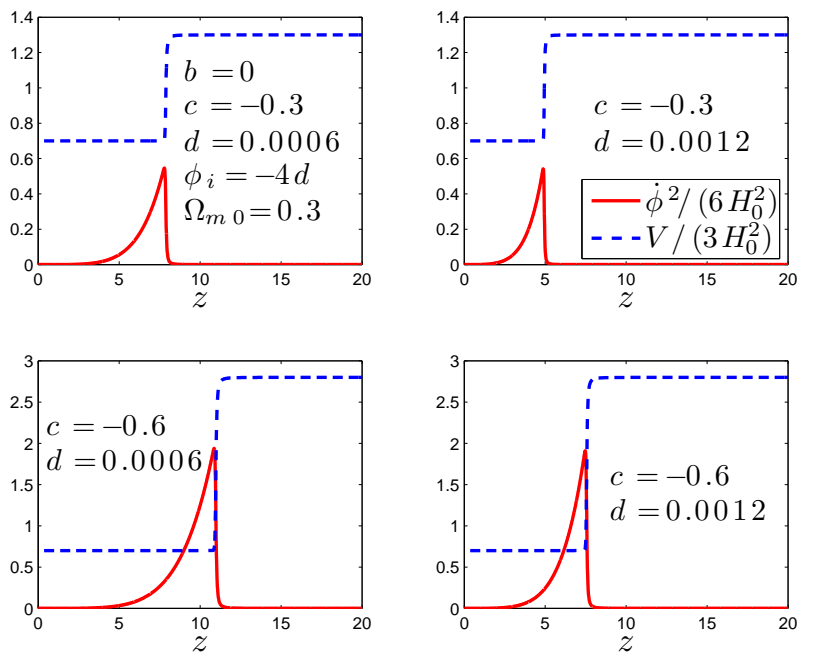

Figure 2: (color online). The rise and fall of the kinetic energy and potential of a stepped quintessence with potential (10) and $f=+1$. Both the kinetic energy and the potential are normalized by $3 H_{0}^{2}$, where $H_{0}$ is the Hubble parameter at $z=0$.

For relatively small $d$, the hyperbolic tangent function is a good smooth analytic approximation to a step function. The parameter $b$ determines the location of step in field space, while $c$ and $d$ control the height and width of step respectively. Based on this model, more complicated ones can be obtained by superposing the steps or replacing the constant $V_{0}$ with other functions. Note here $c$ is a dimensionless parameter, but $b$ and $d$ are in unit of reduced Planck mass $M_{p l}=1 / \sqrt{8 \pi G_{N}}$. However, in our simulation and figures, we will simply set $M_{p l}=1$.
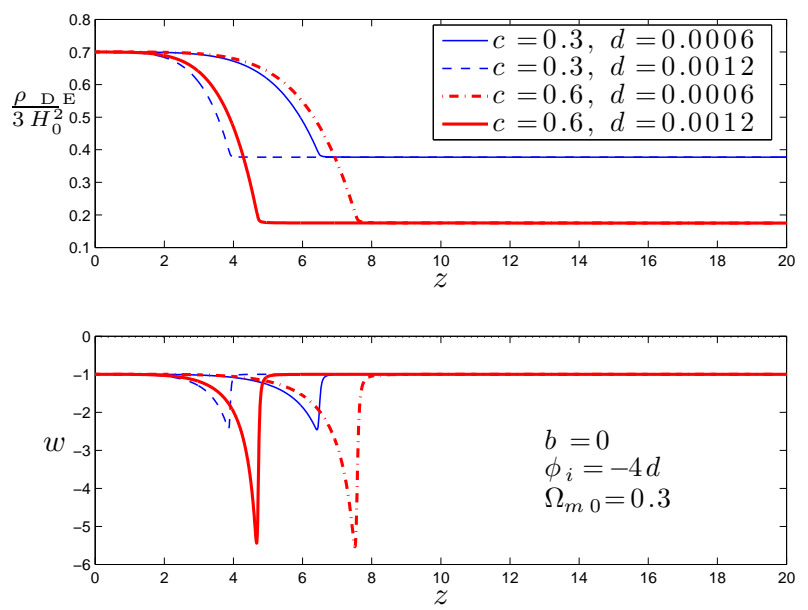

Figure 3: (color online). For stepped phantom with potential (10) and $f=-1$. The dark energy density climbs up a step, while EOS parameter has a dip nearby. Similarly to the stepped quintessence, the amplitude and location of the steps and dips are tunable as we change the model parameters.

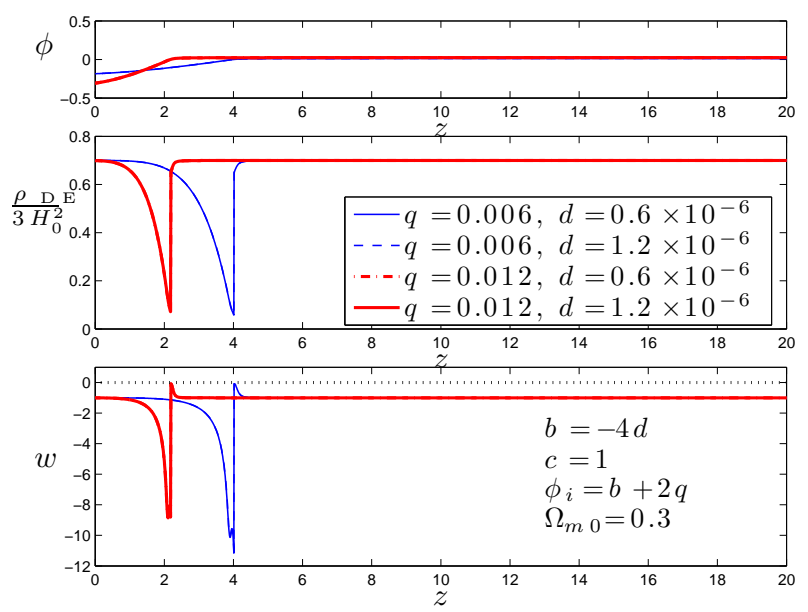

Figure 4: (color online). The evolution of the scalar field, dark energy density and EOS parameter for the stepped quintom model (13). In contrast with previous figures, a small change in parameter $d$ does not modify the evolution curves significantly.

We study this potential in the context of quintessence with $f=+1$ and phantom with $f=-1$ respectively. Since $\Omega_{\mathrm{DE} 0}+\Omega_{m 0}=1$ in a flat universe with negligible ordinary matter, it is easy to prove $V_{0}=3 H_{0}^{2}(1-$ $\left.\Omega_{m 0}\right) /(1 \pm|c|)$ for quintessence (lower sign) and phantom (upper sign). The simulation results for stepped quintessence are shown in figures 1 and 2 . The results for phantom are depicted in figure 3. In plotting them we have chosen the step location $b=0$, and the initial value of $\phi$ is chosen as $\phi_{i}=-4 d$ at redshift $z_{i}=20$. From the figures we can see a step in the energy density. Quintessence falls down the step but phantom climbs up. 
Although the location of step in field space is fixed by $b=0$, its location in redshift space is dependent of $c$ and $d$. At the same location there is a feature (a bump for quintessence or a dip for phantom) in curves of kinetic energy and EOS parameter. Amplitudes of steps and features are determined mainly by parameter $c$. The initial value $\phi_{i}$ of the scalar field resides well in the flat region of the potential because $\tanh (-4) \simeq-0.999$. If we increase the absolute value of $\phi_{i}$, the steps and features will shift to lower redshift region.

From figures 2 and 3, one can roughly read the location $z_{T}$ and width $\Delta_{T}$ of the step as well as the initial density $\rho_{i}$ and final density $\rho_{f}$ of the dark energy. In data analysis, it is useful to capture these properties by parameterizing the dark energy density as

$$
\begin{aligned}
\rho & =\rho_{i}+\frac{\rho_{f}-\rho_{i}}{1+\exp \left(\frac{z-z_{T}}{\Delta_{T}}\right)} \\
& =\frac{\rho_{i}+\rho_{f}}{2}+\frac{\rho_{i}-\rho_{f}}{2} \tanh \left(\frac{z-z_{T}}{2 \Delta_{T}}\right) .
\end{aligned}
$$

This parametrization is applicable when the dark energy changes from $\rho_{i}$ to $\rho_{f}$ near the redshift $z_{T}$. Assuming the dark energy is decoupled with other ingredients, we have the continuity equation $\dot{\rho}+3 H(\rho+p)=0$ or equivalently $(\ln \rho)^{\prime}=-3(1+w)$. Corresponding to (11), it gives

$$
w=\frac{(1+z)\left(\rho_{i}-\rho_{f}\right) \exp \left(\frac{z-z_{T}}{\Delta_{T}}\right)}{3 \Delta_{T}\left[1+\exp \left(\frac{z-z_{T}}{\Delta_{T}}\right)\right]\left[\rho_{f}+\rho_{i} \exp \left(\frac{z-z_{T}}{\Delta_{T}}\right)\right]}-1 .
$$

One may check that the function gives rise to a bump if $\rho_{i}>\rho_{f}$ or a dip when $\rho_{i}<\rho_{f}$, in agreement with figures 1 and 3 .

\section{B. Steps in the Kinetic Term}

Having discussed the steps in the dark energy potential, we discuss what will happen if there is a step in the coefficient $f(\phi)$ of the kinetic term. It is interesting to study a new model

$$
\begin{aligned}
f(\phi) & =c \tanh \left(\frac{\phi-b}{d}\right), \\
V(\phi) & =V_{0}\left[1+\tanh \left(\frac{\phi^{2}}{q^{2}}\right)\right] .
\end{aligned}
$$

In favor of the fact that $\Omega_{\mathrm{DE} 0}+\Omega_{m 0}=1$, we can set $V_{0}=3 H_{0}^{2}\left(1-\Omega_{m 0}\right) / 2$. In the potential $V(\phi)$ there is a deep dip, whose depth has been fixed. The coefficient $f(\phi)$ is non-trivial. It has a step located at $\phi=b$ with amplitude $c$ and width $d$. Again $c$ is a dimensionless parameter, while $b$ and $d$ are in unit of reduced Planck mass $M_{p l}$. As mentioned before, we set $M_{p l}=1$ in our simulation and figures.

The idea is to settle the potential bottom $\phi=0$ in the region $f>0$ by ensuring $c \tanh (-b / d)>0$. Given an appropriate initial condition, the scalar first rolls down to the bottom of potential and then moves up, getting closer and closer to the point $\phi=b$. However, this model still requires some fine-tuning, because it is not always possible for the scalar field to pass the sign-inversion point of $f(\phi)$ after leaving the bottom of potential. We fine-tune the parameters, and then numerically evolve the equation of motion (6). According to the simulation results in figure 4 , both the density and the EOS parameter of dark energy decrease near the sign-inversion point. In particular, the EOS parameter jumps down abruptly from $w>-1$ to $w \ll-1$. This is a simple way to cross the EOS barrier $w=-1$. To make it we need only one real scalar field, completely relying on its own dynamics. However, one should not regard (13) as more than a toy model. There is an infamous instability in any model with a wrong-signed kinetic term, e.g., in the phantom model [5]. We suspect the stepped quintom model here suffers from the same problem. Various issues on this class of model were explored in $[8,9,10]$.

\section{Steps in the EOS Parameter}

If there is a step in the EOS parameter, one usually parameterizes $w$ in a form [1] similar to (11). However, for building a model later, we parameterize it in an alternative way,

$$
w=w_{i}+\frac{w_{f}-w_{i}}{1+\left(\frac{1+z}{1+z_{T}}\right)^{\lambda}}=w_{i}+\frac{w_{f}-w_{i}}{1+\left(\frac{a_{T}}{a}\right)^{\lambda}},
$$

where $\lambda \gg 1$. With this form, the continuity equation can be integrated out as

$$
\begin{aligned}
\rho & =\rho_{T}\left(\frac{a_{T}}{a}\right)^{3\left(1+w_{i}\right)}\left[\frac{1}{2}+\frac{1}{2}\left(\frac{a}{a_{T}}\right)^{\lambda}\right]^{3\left(w_{i}-w_{f}\right) / \lambda} \\
& =\rho_{T}\left(\frac{a_{T}}{a}\right)^{3\left(1+w_{f}\right)}\left[\frac{1}{2}+\frac{1}{2}\left(\frac{a_{T}}{a}\right)^{\lambda}\right]^{3\left(w_{i}-w_{f}\right) / \lambda}
\end{aligned}
$$

Note there is a duality $w_{i} \leftrightarrow w_{f}, \lambda \leftrightarrow-\lambda$ in (14). We are most interested in the following special cases:

- $w_{i}=-1$; Now the EOS takes the form

$$
\frac{p}{\rho}=w_{f}-\frac{1+w_{f}}{2}\left(\frac{\rho}{\rho_{T}}\right)^{\lambda /\left[3\left(1+w_{f}\right)\right]} .
$$

- $w_{f}=-1$. In this case, we reexpress the EOS as

$$
\frac{p}{\rho}=w_{i}-\frac{1+w_{i}}{2}\left(\frac{\rho_{T}}{\rho}\right)^{\lambda /\left[3\left(1+w_{i}\right)\right]} .
$$

The equations of state (16) and (17) can be casted into a unified form

$$
p=\rho\left(w_{c}-A \rho^{\alpha}\right),
$$




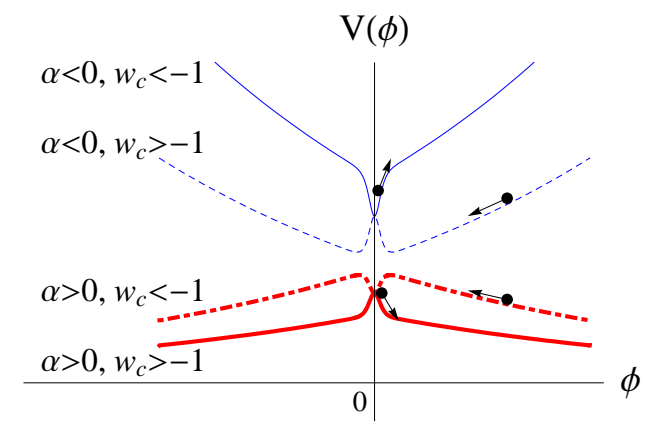

Figure 5: (color online). A rough illustration of potential (20) with different parameter choices. The arrows indicate evolving directions of the dark energy field. For example, when $\alpha<0$ and $w_{c}<-1$, the potential is illustrated by the thin solid blue line, and the scalar field rolls up the potential from the bottom $\phi=0$ to $\phi \rightarrow \pm \infty$, while the EOS parameter $w$ evolves from -1 to $w_{c}$ asymptotically. The dashed blue line corresponds to the potential with $\alpha<0$ and $w_{c}>-1$, in which case the scalar field rolls down the potential from $\phi \rightarrow \pm \infty$ to one of the local minimum point, and the EOS parameter $w$ evolves form $w_{c}$ to -1 .

from which one can integrate the continuity equation to give

$$
\rho^{\alpha}=\frac{1+w_{c}}{A+B a^{3 \alpha\left(1+w_{c}\right)}}=\frac{1+w_{c}}{A\left[1+\left(\frac{a}{a_{T}}\right)^{3 \alpha\left(1+w_{c}\right)}\right]} .
$$

It is time for us to reconstruct the potential of (10) from (14) in a universe dominated by dark energy. To do this, we assume $f(\phi)$ is a nonzero constant and $\rho_{m}=0$, $w_{c} \neq-1$. For details on reconstructing the potential of scalar dark energy, please refer to [11, 12, 13, 14]. Making use of equations (2), (3), (18) and (19), we finally obtain

$$
\begin{aligned}
& a^{3 \alpha\left(1+w_{c}\right)}=\frac{A}{B} \sinh ^{2}\left(\frac{\phi}{\phi_{c}}\right), \\
& V(\phi)=\frac{V_{c}}{2} \cosh ^{-2 / \alpha}\left(\frac{\phi}{\phi_{c}}\right)\left[2-\left(1+w_{c}\right) \tanh ^{2}\left(\frac{\phi}{\phi_{c}}\right)\right]
\end{aligned}
$$

[1] B. A. Bassett, M. Kunz, J. Silk and C. Ungarelli, Mon. Not. Roy. Astron. Soc. 336, 1217 (2002) arXiv:astro-ph/0203383.

[2] Q. G. Huang, M. Li, X. D. Li and S. Wang, arXiv:0905.0797 [astro-ph.CO].

[3] J. A. Adams, B. Cresswell and R. Easther, Phys. Rev. D 64, 123514 (2001) arXiv:astro-ph/0102236.

[4] I. Zlatev, L. M. Wang and P. J. Steinhardt, Phys. Rev. Lett. 82, 896 (1999) arXiv:astro-ph/9807002.

[5] R. R. Caldwell, Phys. Lett. B 545, 23 (2002) arXiv:astro-ph/9908168.

[6] B. Feng, X. L. Wang and X. M. Zhang, Phys. Lett. B 607, 35 (2005) arXiv:astro-ph/0404224.

[7] M. J. Mortonson, W. $\mathrm{Hu}$ and D. Huterer, with $\phi_{c}^{-1}=\alpha \sqrt{6 \pi G_{N} f\left(1+w_{c}\right)}$ and $V_{c}=[(1+$ $\left.\left.w_{c}\right) / A\right]^{1 / \alpha}$. For various choices of parameters, the shape of this potential is illustrated in figure 5. The EOS parameter $w \rightarrow w_{c}$ asymptotically as $\phi \rightarrow \pm \infty$. Near the bottom or top of the potential, it approaches -1 . The field rolls down the potential as a quintessence if $w_{c}>-1$, but rolls up as a phantom when $w_{c}<-1$. We impose $\left|3 \alpha\left(1+w_{c}\right)\right| \gg 1$ by hand to accomplish the sudden EOS transition (14).

\section{DISCUSSION}

Treating dark energy as a scalar field, we explicitly modeled steps in its potential, kinetic term, density and EOS, and thus provided a workable mechanism to explain the previously and recently claimed dark energy transitions. To arrive at a realistic model, more experimental and theoretical efforts are needed in the future. When this work was near completion, a related paper 7] appeared, where they arrived at models with an implicit potential similar to the shape of $V(\phi)$ in(13). As a partial list, some previous papers relevant to our work are [15, 16, 17, 18, 19, 20, 21].

\section{Acknowledgments}

This work is supported by the China Postdoctoral Science Foundation. We are grateful to Tong Li, Wei Liao for discussions and Qinyan Tan for help in programme.

arXiv:0908.1408 [astro-ph.CO].

[8] A. Vikman, Phys. Rev. D 71, 023515 (2005) arXiv:astro-ph/0407107.

[9] S. Nojiri and S. D. Odintsov, Gen. Rel. Grav. 38, 1285 (2006) arXiv:hep-th/0506212.

[10] J. Q. Xia, Y. F. Cai, T. T. Qiu, G. B. Zhao and X. Zhang, Int. J. Mod. Phys. D 17, 1229 (2008) arXiv:astro-ph/0703202.

[11] T. D. Saini, S. Raychaudhury, V. Sahni and A. A. Starobinsky, Phys. Rev. Lett. 85, 1162 (2000) arXiv:astro-ph/9910231.

[12] V. Sahni and A. Starobinsky, Int. J. Mod. Phys. D 15, 2105 (2006) arXiv:astro-ph/0610026.

[13] S. Capozziello, S. Nojiri and S. D. Odintsov, Phys. Lett. 
B 632, 597 (2006) arXiv:hep-th/0507182.

[14] E. Elizalde, S. Nojiri, S. D. Odintsov, D. Saez-Gomez and V. Faraoni, Phys. Rev. D 77, 106005 (2008) arXiv:0803.1311 [hep-th]].

[15] S. Y. Zhou, Phys. Lett. B 660, 7 (2008) arXiv:0705.1577 [astro-ph]].

[16] S. Capozziello, S. Nojiri and S. D. Odintsov, Phys. Lett. B 634, 93 (2006) arXiv:hep-th/0512118.

[17] S. Nojiri and S. D. Odintsov, Phys. Lett. B 637, 139 (2006) arXiv:hep-th/0603062.
[18] S. Nojiri, S. D. Odintsov and H. Stefancic, Phys. Rev. D 74, 086009 (2006) arXiv:hep-th/0608168.

[19] S. Nojiri, S. D. Odintsov and S. Tsujikawa, Phys. Rev. D 71, 063004 (2005) arXiv:hep-th/0501025.

[20] M. Szydlowski, O. Hrycyna and A. Krawiec, JCAP 0706, 010 (2007) arXiv:hep-th/0608219.

[21] O. Hrycyna and M. Szydlowski, arXiv:0906.0335 [astroph.CO]. 